\title{
Questões Éticas da Pesquisa em Psicologia do Desenvolvimento
}

\author{
Ethical Issues In Research On \\ Developmental Psychology
}

Cuestiones Éticas De La Investigación

En Psicología Del Desarrollo
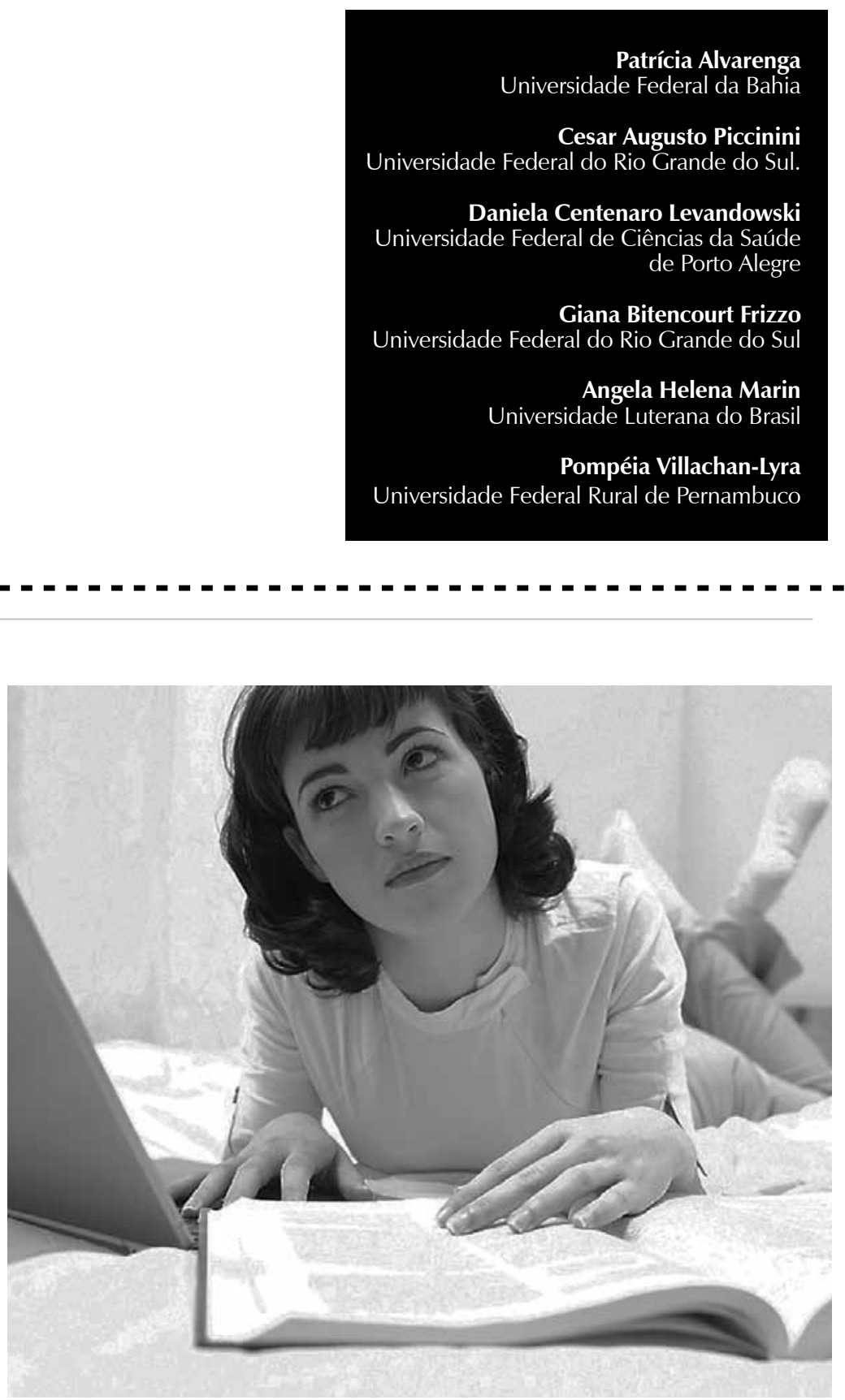
Resumo: A pesquisa em Psicologia do desenvolvimento impõe aos pesquisadores desafios e dilemas éticos peculiares, especialmente em se tratando de investigações que envolvem crianças e adolescentes. $\mathrm{O}$ presente estudo tem o objetivo de apresentar e discutir algumas dessas questões éticas, como a obtenção do Consentimento Livre e Esclarecido, o encaminhamento dos participantes para tratamento e a devolução de avaliações, o uso de instrumentos que não foram validados, os dilemas relativos à sexualidade e saúde de adolescentes e as questões éticas referentes à realização de estudos de caso. Os questionamentos e as reflexões propostos basearam-se, essencialmente, na prática de pesquisa dos próprios autores, em grande parte respaldada pela legislação brasileira e pela literatura sobre ética da pesquisa com seres humanos e da pesquisa em Psicologia. Contudo, constatou-se a existência de várias lacunas na literatura sobre o tema, que indicam a necessidade de aprofundamento do debate sobre a ética na pesquisa em Psicologia do desenvolvimento.

Palavras-chave: Ética. Psicologia do desenvolvimento. Crianças. Adolescentes.

Abstract: Research in developmental psychology imposes challenges and particular ethical dilemmas for researchers, especially when children and adolescents are concerned. The present study aims to present and discuss some of those ethical issues such as obtaining the informed consent, the participants' referral to treatment and the evaluations, the use of instruments that were not validated, dilemmas regarding adolescents' sexuality and health, and ethical issues concerning carrying out case studies. The questions and reflections proposed were based, essentially, on the authors' research practice, largely backed by the Brazilian legislation and by the literature on research ethics with human beings and on Psychology research. However, several gaps were observed in the literature on the theme, that indicate the need for further debate on research ethics in developmental psychology.

Key words: Ethics. Developmental psychology. Children. Adolescents.

Resumen: La investigación en Psicología del desarrollo les impone a los encuestadores desafíos y dilemas éticos peculiares, especialmente cuando se trata de investigaciones que involucran a niños y adolescentes. El presente estudio tiene el objetivo de presentar y discutir algunas de esas cuestiones éticas, como la obtención del Consentimiento Libre y Aclarado, el encaminamiento de los participantes para tratamiento y la devolución de evaluaciones, el uso de instrumentos que no fueron validados, los dilemas relativos a la sexualidad y salud de adolescentes y las cuestiones éticas referentes a la realización de estudios de caso. Los cuestionamientos y las reflexiones propuestos se basaron, esencialmente, en la práctica de investigación de los propios autores, en gran parte respaldada por la legislación brasileña y por la literatura sobre ética de la investigación con seres humanos y de la investigación en Psicología. Sin embargo, se constató la existencia de varios vacíos en la literatura sobre el tema, lo que indica la necesidad de profundizarse en el debate sobre la ética en la investigación en la Psicología del desarrollo.

Palabras clave: Ética. Psicología del desarrollo. Niños. Adolescentes.

Os princípios éticos da pesquisa visam a proteger os direitos, a dignidade e o bemestar dos participantes (Barker, Pistrang, \& Eliott, 1995). Essas preocupações éticas cresceram muito a partir do século passado, devido às atrocidades cometidas com fins supostamente científicos nos campos de concentração, e, no contexto da Psicologia, foram particularmente impulsionadas por pesquisas sobre indução de estresse e por investigações que envolviam a omissão de informações na área da Psicologia social nos anos 50 e 60. O movimento de direitos civis, ocorrido nos anos 60 e 70, também aumentou a sensibilidade dos psicólogos e pesquisadores em relação à ética (Barker et al., 1995). Nesse período, em uma tentativa de maior controle sobre as investigações científicas, surgiram os primeiros códigos de ética internacionais, que contaram com revisões no decorrer do tempo.

Os debates sobre diretrizes e normas éticas para a pesquisa com seres humanos e, particularmente, para a pesquisa em Psicologia, avançaram muito nos últimos anos, inclusive no Brasil. Atualmente, a legislação prevê que os participantes tenham garantia de proteção contra uma série de constrangimentos e possíveis consequências indesejáveis decorrentes da participação em pesquisas, e que recebam informações 
claras a respeito de todo o processo a que serão submetidos. Do mesmo modo, os pesquisadores contam com um conjunto de normas bem estabelecidas, que contempla grande parte das implicações éticas com que poderão se deparar no planejamento e na execução de diferentes tipos de investigação, o que também os protege de eventuais litígios.

Contudo, a prática em pesquisa, como um processo dinâmico, apresenta continuamente novas questões que ainda não foram contempladas pela legislação vigente, e que, por vezes, acabam se constituindo em obstáculos para a sua realização (Koller, 2008). Isso pode ser evidenciado pelos inúmeros códigos de ética que foram se sucedendo nas últimas décadas e que, com certeza, continuarão sendo atualizados. Por outro lado, segundo Miller (2007), as diretrizes éticas costumam ser bastante genéricas, exigindo dos pesquisadores e dos Comitês de Ética uma reflexão cuidadosa a respeito de como elas podem ser aplicadas a casos especiais.

Considerando as especificidades do campo da Psicologia do desenvolvimento, caracterizado fundamentalmente por estudos com crianças e adolescentes, o presente estudo tem como objetivo apresentar e refletir sobre alguns desses desafios e dilemas éticos com os quais os pesquisadores dessa área têm se deparado. Muitas vezes, esses desafios são de difícil manejo, como, por exemplo, a obtenção do Termo de Consentimento Livre e Esclarecido, o encaminhamento dos participantes para tratamento quando necessário e a devolução de avaliações que requerem encaminhamentos. Outras questões importantes que serão examinadas referem-se ao uso de instrumentos que não foram validados, de questões que envolvem a sexualidade e a saúde de adolescentes e a realização de estudos de caso, que podem expor os participantes, especialmente quando a pesquisa contempla algum tipo de intervenção clínica. Todas essas situações têm sido vivenciadas na prática de pesquisa dos autores do presente estudo e são aqui discutidas à luz da legislação brasileira sobre ética na pesquisa e, mais especificamente, sobre ética da pesquisa em Psicologia. Esperase que essa reflexão possa contribuir para enriquecer o debate sobre o tema bem como para o processo de tomada de decisão dos pesquisadores da área, diante dos dilemas éticos cotidianos impostos pela práxis da pesquisa.

\section{O Termo de Consentimento Livre e Esclarecido em pesquisas com crianças e adolescentes}

As Diretrizes e Normas Regulamentadoras de Pesquisas Envolvendo Seres Humanos, do Conselho Nacional de Saúde (Resolução CNS no 196/96), ora vigentes no Brasil, e complementadas pela Resolução $n^{\circ}$ 016/2000, do Conselho Federal de Psicologia, indicam que todo projeto de pesquisa que envolver seres humanos deve incluir um Termo de Consentimento Livre e Esclarecido (TCLE). Através dele, o participante fica ciente do que acontecerá no estudo e recebe informações que podem afetar a sua decisão de participar (Goldim, Pithan, Oliveira, \& Raymundo, 2003).

Uma exceção à exigência do TCLE referese a pesquisas que envolvam unicamente a consulta a prontuários de pacientes ou a bases de dados como fontes de informação. Mesmo assim, algumas instituições têm utilizado um Termo de Compromisso para Utilização de Dados, como forma de disciplinar o acesso às informações de pacientes (Francisconi \& Goldim, 1998). Esse documento também pode ser usado quando os participantes da pesquisa não puderem ser contactados ou localizados. 
A obrigatoriedade do TCLE incita questionamentos e desafios para a prática de algumas pesquisas, especialmente aquelas concernentes a populações consideradas incapazes de exercer pessoalmente atos da vida civil, conforme o Código Civil Brasileiro (Brasil, Lei $\left.n^{\circ} 10.406,2002\right)$, a saber: menores de 16 anos, pessoas que, por enfermidade ou deficiência mental, não tiverem o necessário discernimento para responsabilizar-se pelos seus atos e aquelas que, mesmo por causa transitória, não puderem exprimir sua vontade. Considerando tais aspectos, faz-se importante discutir as questões relativas ao TCLE nas pesquisas relacionadas à Psicologia do desenvolvimento, que envolvem crianças e adolescentes.

Mais do que um documento, o TCLE deve ser entendido como um processo por meio do qual a pessoa recebe uma explicação minuciosa sobre os procedimentos da pesquisa, compreende a informação, atua voluntariamente para, finalmente, consentir ou não em participar do estudo (Goldim, 1997). Portanto, há quatro elementos necessários para que esse documento seja considerado válido: o fornecimento de informação, a compreensão, a voluntariedade e o consentimento propriamente dito.

No que diz respeito ao fornecimento de informação e a sua compreensão, destacase que é importante utilizar uma linguagem acessível, evitando o uso de terminologias técnicas e de difícil entendimento para a pessoa que deverá assinar o documento, além de evitar o uso de frases e de parágrafos muito extensos. É importante esclarecer sobre a pesquisa, assinalando seus objetivos, sua justificativa e os procedimentos que serão utilizados. Caso haja, por questões metodológicas, a necessidade de omitir informações, essa situação deve ser apresentada e justificada ao Comitê de Ética em Pesquisa. Os riscos e desconfortos, assim como os benefícios e o acompanhamento assistencial, quando necessário, também devem ser descritos, incluindo o tempo previsto para a participação no estudo. Ainda, faz-se necessário constar a disponibilidade do pesquisador para responder as dúvidas a qualquer momento da realização do estudo e a afirmação de que o participante pode retirar seu consentimento quando assim o desejar. Goldim et al. (2003) salientam que, quando esses aspectos não forem considerados, o texto pode ser de difícil compreensão, além de poder levar a interpretações ambíguas devido à falta, ao tipo ou à incoerência das informações.

A voluntariedade e o consentimento, por sua vez, requerem que não haja influências controladoras exercidas por outras pessoas, o que implica autonomia do participante para consentir. Nesse sentido, questiona-se o consentimento de crianças e adolescentes quando essa é a população- alvo das pesquisas. Legalmente, a decisão sobre a participação de menores de idade em projetos de pesquisa cabe à família ou a seus representantes legais, porque são considerados incapazes de exercer pessoalmente atos da vida civil. No entanto, ninguém pode exercer o direito de consentir por outra pessoa, pois essa é uma ação individual e indelegável. Em função disso, considera-se que tanto a criança ou o adolescente quanto a família ou seu representante legal devem participar do processo de obtenção do consentimento, até porque a capacidade para entender e decidir não tem relação direta com a idade cronológica (Goldim, 2006).

Nessa mesma direção, a compreensão e a autonomia de adolescentes têm sido questionadas nas pesquisas que abordam, por exemplo, o processo gestacional e a maternidade. Sabe-se que a gravidez na adolescência não garante automaticamente à jovem o status de adulta, e que a gestação pode ter decorrido justamente de sua imaturidade biopsicossocial, dentre outros 
fatores (Figueiredo, 2001). Entretanto, uma vez que a gestação é uma realidade e que a jovem assume a maternidade, tornando-se, ela própria, responsável legal por sua criança, por que não considerá-la apta a avaliar uma proposta de pesquisa e a viabilidade e a adequação de sua participação? Por que a necessidade de solicitar aos seus responsáveis legais o consentimento?

Conforme apontam alguns estudos (Flicker \& Guta, 2008; Guariglia, Bento, \& Hardy, 2006; Santelli, Rogers, Rosenfeld, DuRant, Dubler, Morreale, English, Lyss, Winberly, \& Schissel, 2003), os próprios adolescentes consideramse aptos a decidir a sua participação em pesquisas, e afirmam tomar essa decisão de modo consciente. Ainda, segundo Guariglia et al. (2006), existe desconhecimento dos pesquisadores a respeito do fato de que, conforme o Código Civil Brasileiro (Brasil, Lei $\mathrm{n}^{\circ}$. 10.406, de 2002), a incapacidade legal de um menor cessa quando ele se casa ou mantém uma união estável. Esse inciso não deveria ser ignorado pelos Comitês de Ética em Pesquisa, que geralmente exigem a assinatura do TCLE por um responsável legal pela adolescente menor de 18 anos (pai, mãe, outro familiar, tutor ou companheiro), mesmo em estudos cujas participantes já mantenham uma relação de casamento/união estável, para os quais, como referido por Guariglia et al., isso não se faria necessário.

Outra dificuldade relativa ao TCLE referese a pesquisas que abordam a sexualidade de adolescentes. Em uma pesquisa com adolescentes soropositivas, por exemplo, entregar aos pais um documento que contenha o título ou o tema da pesquisa, bem como as informações referentes a ela, pode infringir o direito à confidencialidade e à privacidade do adolescente. Nesse sentido, em se tratando de temas delicados de investigação, como o aborto ou a atividade sexual, conforme as Diretrizes da ONU referentes à pesquisa em Saúde Reprodutiva envolvendo adolescentes (Organização das Nações Unidas, 2011), a confidencialidade do adolescente pode ser preservada. Essa medida estaria pautada no princípio bioético da beneficência, por facilitar a participação de grupos vulneráveis, que teriam acesso aos benefícios diretos e indiretos do estudo. Por outro lado, a necessidade de tal autorização dos pais pode impedir a pesquisa de dar voz a adolescentes que, diante da não autorização de seus responsáveis, não participarão do estudo (Flicker \& Guta, 2008). Percebe-se que tal consideração não tem sido adotada pelos Comitês de Ética em Pesquisa, que, ao primar pela proteção desse grupo duplamente vulnerável (por exemplo, pela idade ou pela soropositividade), têm se mostrado ainda mais rigorosos e criteriosos na avaliação das pesquisas propostas a esse público.

Como visto, é necessário que o processo de obtenção do consentimento abarque mais do que a simples suposição de autonomia dos participantes, sejam eles crianças, sejam adolescentes, visando a obter a sua autorização, pois todos, independentemente de sua idade ou capacidade, merecem respeito e cuidado com as informações a eles pertinentes. Nesse sentido, concorda-se com Goldim (2006), que afirma que o TCLE deve ser entendido a partir de uma interação entre pesquisador-pesquisado, o que implica responsabilizar-se pelo outro e por si mesmo.

\section{A devolução de avaliações de crianças e adolescentes em estudos longitudinais}

Estudos de caráter longitudinal permitem que a amostra de participantes seja acompanhada por um determinado período de tempo, ao longo do qual preditores de um fenômeno de interesse podem ser avaliados e relacionados entre si. Assim, processos desenvolvimentais podem ser acompanhados à medida que evoluem (Miller, 2007). Contudo, a exemplo 
de outros tipos de delineamentos empregados na pesquisa em Psicologia, os estudos longitudinais também impõem alguns desafios ao investigador. Além dos desafios de caráter metodológico e operacional, questões e dilemas éticos particulares também podem ser suscitados nesse tipo de investigação.

Muitos estudos longitudinais têm por objetivo investigar fenômenos relacionados à psicopatologia e ao desenvolvimento atípico (referências omitidas para preservar a autoria). Nesses casos, é comum a prática de selecionar amostra considerada de risco para o desenvolvimento de determinado fenômeno ou processo desenvolvimental, com a expectativa de que tal padrão surja entre os participantes e possa ser acompanhado e investigado ao longo de um determinado período. A própria concepção de um projeto de pesquisa com esse caráter pode constituir o primeiro dilema ético, afinal, o estudo concebido prevê e depende do desenvolvimento de alguma característica atípica na amostra, cujos fatores predisponentes foram cuidadosamente previstos pelo pesquisador para assegurar sua ocorrência, sem a qual o estudo não seria possível. De qualquer modo, embora tal dilema possa ocorrer, ele não parece impor ao pesquisador a necessidade de algum tipo de ação reparadora antecipatória, visto que, no momento da concepção da pesquisa e da seleção da amostra de risco, o fenômeno (que constitui o objeto de estudo) ainda não se fazia presente.

Contudo, ao ser confrontado com os primeiros indicadores de algum padrão de desenvolvimento atípico ou disfuncional, o pesquisador se depara com a seguinte questão: os participantes do estudo devem receber a devolução da avaliação que indicou a presença do padrão atípico? Eles devem ser alertados para os prejuízos que tal fenômeno pode acarretar? Considerando que, para os participantes, os indicadores do padrão atípico de desenvolvimento não são percebidos como uma queixa ou problema que necessita de intervenção, como abordar o encaminhamento para algum tipo de tratamento? É importante atentar também para o fato de que, ao intervir ou encaminhar os participantes para tratamento, o pesquisador poderá inviabilizar a continuidade do seu estudo longitudinal, na medida em que o tratamento pode fazer com que o fenômeno investigado desapareça ou se modifique.

Dessen, Avelar e Dias (1998) abordam as implicações da devolução de resultados em estudos longitudinais e defendem a possibilidade de se fazer isso só após a conclusão da pesquisa. Nesses casos, as autoras sugerem que sejam realizadas entrevistas individuais para que os participantes possam falar sobre sua experiência de fazer parte do estudo, sem que o pesquisador revele os resultados da pesquisa. Por outro lado, os princípios éticos para a pesquisa com crianças da Society for Research in Child Development (1990 como citado em Miller, 2007), embora não tratem dessa questão específica, preconizam a não retenção de informações que possam colocar em risco o desenvolvimento e o bem-estar da criança, o que inclui a devolução cuidadosa dos resultados para os participantes. Ao mesmo tempo, tais princípios recomendam cautela por parte do pesquisador ao reportar os achados e ao fazer devoluções de caráter avaliativo ou dar qualquer tipo de aconselhamento aos pais. De qualquer modo, essas diretrizes sugerem que indicadores de padrões de desenvolvimento atípico, constatados nas avaliações realizadas como procedimento da pesquisa, deveriam ser notificados aos participantes ou aos seus responsáveis.

Outra questão polêmica diz respeito à forma de fazer esse tipo de devolução. Não há 
diretrizes claras na literatura a respeito de como fazê-lo. Uma possibilidade é a de que o pesquisador, além de informar os resultados da avaliação e apontar a necessidade e os benefícios do tratamento, esteja disponível para conversar com os participantes ou responsáveis, individualmente ou em grupo, prestando esclarecimentos. Nesse caso, considerando os resultados controversos e inconclusivos de grande parte das pesquisas, é interessante refletir sobre a postura do pesquisador nesse tipo de situação. Talvez uma alternativa seja mostrar claramente as dúvidas e as incertezas que circundam aquele objeto de pesquisa, até mesmo em razão da idade dos participantes, procurando não ser taxativo e excessivamente fatalista ao indicar os prejuízos que poderão decorrer do não tratamento da condição-problema. É claro que este será mais um grande desafio para o pesquisador, já que a linguagem a ser utilizada para referir-se a essas lacunas e incertezas deve dar conta das demandas dos participantes.

Após a devolução, é natural que os participantes da pesquisa manifestem interesse em receber uma avaliação mais detalhada da situação da criança ou do adolescente, ou mesmo queiram buscar tratamento para o problema. Assim, é aconselhável que, na medida do possível, o pesquisador possa garantir pelo menos o encaminhamento desses casos. A condição de aflição e de desamparo gerada nos pais pela devolução pode se tornar ainda mais angustiante para populações de baixa renda, que geralmente dispõem de pouca informação e de recursos emocionais e comportamentais mais precários para lidar com o problema, além de terem pouco acesso a serviços de atenção à saúde de qualidade. Nesses casos, o pesquisador poderia dispor de uma lista razoável de locais de atendimento gratuito ou com valores acessíveis e fazer um encaminhamento cuidadoso, que incluísse todas as informações e orientações necessárias para que os participantes tivessem sucesso na busca de auxílio. Outra conduta que pode ser adotada é a de acompanhar, por meio de contatos telefônicos ou de outras formas de comunicação com a família, o processo de busca pelo tratamento, pelo menos até que a primeira consulta seja realizada ou até que o primeiro contato com o local de atendimento tenha sido feito. Também tem sido prática de alguns grupos de pesquisa disponibilizar algumas sessões de atendimento psicológico pelos próprios membros da equipe, com o objetivo de fazer uma avaliação mais cuidadosa da situação encontrada e da demanda do participante para atendimento, facilitando o encaminhamento do caso para alguma modalidade de atendimento mais sistemático.

Por fim, após a devolução e os devidos encaminhamentos, o pesquisador será confrontado com novas questões, agora de cunho metodológico, mais do que ético: que destino terão, dentro da pesquisa, os participantes que foram encaminhados e que estão em tratamento? E quanto aos participantes que, mesmo sabendo dos resultados da avaliação, preferiram não buscar atendimento? Pensa-se que, após ter resolvido de forma responsável e sensível os dilemas éticos relacionados a sua escolha metodológica, é possível que o pesquisador se beneficie dessa atitude, pela possibilidade que terá de avaliar os efeitos do tratamento sobre um grupo de participantes ou de um pequeno número de casos. Essa nova alternativa metodológica poderá enriquecer o desenho do estudo original e permitir a discussão de novas variáveis associadas ao fenômeno investigado.

\section{Estudos sobre a sexualidade e a saúde dos adolescentes}

A pesquisa com adolescentes impõe desafios peculiares, relacionados às características 
dessa faixa etária, bem como alguns dilemas éticos, especialmente no que tange à saúde e à sexualidade. Em se tratando da gestação na adolescência, por exemplo, uma questão difícil com a qual o pesquisador dessa área se depara é a grande diferença de idade, por vezes encontrada entre gestantes e mães adolescentes e seus parceiros, que leva ao seguinte questionamento: trata-se de caso para denúncia aos órgãos competentes ou não? Em alguns casos, a diferença pode chegar a quase 30 anos, como encontrado em estudos realizados na cidade de Porto Alegre (referência omitida para preservar autoria) e na região do Vale dos Sinos, no Rio Grande do Sul (referência omitida para preservar autoria).

Para o Código Penal Brasileiro, um quadro como esse poderia caracterizar uma situação de crime (estupro), tendo em vista a idade da adolescente, o que permitiria inclusive a realização de um aborto. Da mesma forma, o Estatuto da Criança e do Adolescente (Brasil, 1990) indica que qualquer situação de violência contra adolescentes deve ser denunciada ao Conselho Tutelar. Entretanto, o que fazer quando o pesquisador não vê o uso da força ou uma aparente submissão da jovem ao seu parceiro bem mais velho? Nesse caso, poder-se-ia falar em abuso, se a própria jovem não sente que está sendo submetida a um relacionamento não consentido? Em que medida a avaliação pessoal das adolescentes acerca de sua condição deve ser respeitada? Ou, como apontam Libório e Castro (2010), seria essa uma situação de perversão, ao invés de uma relação amorosa, que, justamente por seu caráter, impediria que a adolescente pudesse refletir criticamente a seu respeito?

Assim, uma situação conjugal como essa deveria ser denunciada ao Conselho Tutelar? Como tomar tal medida, diante de uma adolescente cuja família e a equipe de saúde não impediram tal situação e para quem a vida conjugal e familiar atual se mostra satisfatória? Para quem denunciar, se a família da adolescente já desistiu da filha que se perdeu, no dizer de Taquette, Vilhena, Silva e Vale (2005)? Ou, como apontam NeivaSilva, Lisboa e Koller (2005), para quem denunciar, quando a família da jovem não se mostra protetora? Que amparo institucional o pesquisador encontra e em que instituições governamentais poderá buscar auxílio para a jovem que, muitas vezes, não conta com sua família? Por outro lado, ao se denunciar essas situações, muitas participantes de estudos sobre gravidez na adolescência, cujos parceiros são bem mais velhos que elas, teriam que eventualmente ser excluídas das amostras desses estudos, como sugerido acima, em referência às questões éticas envolvidas em investigações longitudinais.

Situações como essa também são conflitivas para profissionais de saúde que lidam com o público adolescente. Entretanto, conforme apontado no estudo de Taquette et al. (2005), a presunção de estupro deixa de existir frente ao conhecimento do profissional acerca de sua não ocorrência, respeitando-se as informações trazidas pela adolescente e realizando-se uma avaliação criteriosa do caso. A partir disso, percebe-se a necessidade de buscar uma flexibilização da atitude a ser tomada pelo pesquisador, o que não significa uma posição omissa, mas sim, de investigação cuidadosa e de respeito à autonomia da adolescente, quando for o caso (Flicker \& Guta, 2008; Holder, 2008; Santelli et al., 2003).

De qualquer forma, pelo imperativo da denúncia decorrente do aparato legal brasileiro, em estudos realizados com essa clientela, nem sempre é possível conhecer mais detalhadamente tal realidade, justamente pelo medo de alguns dos parceiros das jovens de que os pesquisadores sejam representantes de órgãos como Conselho Tutelar ou até mesmo da polícia. Isso, por um lado, indica a consciência desses homens a respeito da estranheza que tal situação conjugal desperta 
socialmente, mas, por outro, aqueles que aceitam participar de pesquisas e, em alguma medida, revelam um pouco de sua vida, transmitem a impressão de não terem nada a esconder na forma como estão conduzindo sua relação conjugal e familiar. Contudo, entende-se que casos como esse são realmente complexos, colocando o pesquisador, conforme Neiva-Silva et al. (2005), frente à obrigatoriedade de denunciar tal situação, concomitantemente ao medo de romper o vínculo de confiança estabelecido com os participantes e contratado por meio do TCLE, bem como de colocar em risco o próprio bem-estar da adolescente, considerando a ausência de apoio familiar e institucional encontrada em alguns casos e a sua própria escolha.

Mais recentemente, em investigação com adolescentes soropositivas (referência omitida para preservar autoria), outro desafio ético surgiu: a revelação da soropositividade para HIV. Taquette et al. (2005) já haviam destacado que isso pode constituir um conflito ético para os profissionais que atendem essa clientela. Como convidar adolescentes para participar de pesquisas que envolvem HIV, quando estão acompanhados por outras pessoas que, porventura, desconhecem sua condição sorológica? No contexto norte-americano, para situações como essa, cogita-se, inclusive, da dispensa de uma autorização formal dos pais para a participação na pesquisa (Flicker \& Guta, 2008; Holder, 2008; Santelli et al., 2003). Também no contexto brasileiro, existem particularidades éticas quando se trata de adolescentes em condições específicas de saúde, como o HIV/AIDS. As Diretrizes dos Centros de Testagem e Aconselhamento em HIV/AIDS do Ministério da Saúde (1999) orientam que o adolescente, após avaliada a sua condição de discernimento, deve ter sua vontade respeitada quanto à revelação do diagnóstico para outras pessoas, o que inclui os familiares. Obviamente, isso não impede que o pesquisador auxilie esse indivíduo a pensar sobre o seu diagnóstico e sobre medidas alternativas à manutenção do segredo (Neiva-Silva et al., 2005). Da mesma forma, estando em serviços que atendem esse público, por mais que se presuma o diagnóstico positivo para HIV, é necessária uma abordagem sensível dos adolescentes, que possibilite questionar o diagnóstico e, em caso positivo, conhecer minimamente a forma de transmissão e o tempo de contaminação. Isso, na maioria das vezes, implica abordar a sua sexualidade.

Esses e outros dilemas éticos complexos que se colocam para o pesquisador da adolescência muitas vezes podem desmotivá-lo, por constituírem empecilhos para a continuidade dos seus estudos. Entretanto, especialmente no que tange à saúde sexual e reprodutiva nessa faixa etária, pensa-se, como Santelli et al. (2003), que os interesses científicos são legítimos, o que justifica os investimentos na área. Cabe ao pesquisador, então, buscar formas de proteger os participantes, a fim de gerar novos conhecimentos e ferramentas de intervenção que promovam maior bem-estar e qualidade de vida para nossos jovens.

\section{O uso de instrumentos não validados: o exemplo da avaliação neuropsicológica no início da vida}

A pesquisa em neuropsicologia do desenvolvimento investiga tanto o curso normal do desenvolvimento neuropsicológico infantil como também busca compreender e intervir em casos de alterações do neurodesenvolvimento cognitivo e comportamental, tais como: transtornos da aprendizagem, transtornos invasivos do desenvolvimento, síndromes genéticas, disfunções neurológicas e lesões encefálicas congênitas ou adquiridas (Hazin, Gomes, \& Lemos, no prelo). Nesse campo de estudo recente, instrumentos de avaliação que deem 
Como apontam Binder e Thompson (1995), em situações de pesquisa, o uso de instrumentos não validados em populações diferentes daquelas para as quais foram desenvolvidos não deve ser considerado antiético, sendo, inclusive, necessário para determinadas populações, como é o caso das crianças muito pequenas. conta da complexidade e da especificidade do desenvolvimento neuropsicológico na infância são escassos em nosso país. Considerando (a) a elaboração de parâmetros de avaliação para populações específicas como, por exemplo, crianças com alterações do neurodesenvolvimento cognitivo e comportamental e (b) a necessidade de desenvolver tais parâmetros conforme as especificidades e peculiaridades regionais, essa carência se torna ainda mais marcante.

Entretanto, ao mesmo tempo em que é clara a escassez de instrumentos, é também marcante a importância do estudo do neurodesenvolvimento na primeira infância, dado o caráter de imaturidade e plasticidade do sistema nervoso central no início da vida, com destaque para a plasticidade cerebral. Esta é concebida como uma mudança adaptativa na estrutura e na função do sistema nervoso, que envolve a possibilidade de um cérebro em desenvolvimento reorganizar seus múltiplos padrões de resposta e conexões em função da experiência e da estimulação, tornando os bebês e crianças pequenas extremamente sensíveis às intervenções ambientais (Muszkat, 2006). Considerando tais características, é de suma importância a realização de pesquisas em neurodesenvolvimento que favoreçam melhor compreensão dos aspectos neuropsicológicos na infância como também a realização de intervenção precoce em casos de alterações no neurodesenvolvimento infantil. Com esse objetivo, é crescente a realização de estudos de casos clínicos com crianças que se suspeita de possuírem essas alterações. Muitas vezes, tais estudos têm sido realizados com base em avaliações que utilizam instrumentos ainda não padronizados e validados para o Brasil, embora estejam consagrados na literatura científica.

Como apontam Binder e Thompson (1995), em situações de pesquisa, o uso de instrumentos não validados em populações diferentes daquelas para as quais foram desenvolvidos não deve ser considerado antiético, sendo, inclusive, necessário para determinadas populações, como é o caso das crianças muito pequenas. No entanto, tal uso deve ser limitado a circunstâncias especiais, e os riscos, benefícios e possíveis prejuízos dessa escolha devem ser considerados com muita cautela pelo pesquisador.

Com esse cenário, mesmo para fins de pesquisa, cabem as seguintes questões: seria legítimo o uso de instrumentos que não são validados, destacando-se que precisamos ser cautelosos quanto às nossas conclusões? Seriam esses dados confiáveis? Seria mais adequado, do ponto de vista ético, evitar o uso desses instrumentos e demandar que eles sejam validados? Enquanto isso, como conduzir as investigações? Ou, por outro lado, devemos continuar usando esses instrumentos, reduzindo nossa culpa por fazê-lo em nome de uma pesquisa ou de que os resultados serão relativizados, já que parâmetros psicométricos para vários instrumentos ainda são inexistentes em nosso país? Essas alternativas não parecem ser as melhores para que se avance no desenvolvimento e no uso de instrumentos de avaliação neuropsicológica, principalmente na infância, área ainda tão carente. Ao mesmo tempo, considerando as características do neurodesenvolvimento na primeira infância anteriormente mencionadas, é urgente e necessária a realização de pesquisas que abordem os transtornos do neurodesenvolvimento, com o objetivo de favorecer uma intervenção cada vez mais eficiente e precoce e com vistas a potencializar as possibilidades de desenvolvimento dessas crianças. Mas, como fazê-lo?

Considerando os princípios bioéticos, Haase (2007, p.194) afirma que a postura ética compatível com a pesquisa em neuropsicologia, de modo geral e, em particular, em Psicologia do desenvolvimento, 
se ancora em uma "filosofia que utiliza a moralidade dos comportamentos em função das suas conseqüências, procurando salvaguardar os interesses das partes envolvidas". Nesse sentido, a pesquisa em Psicologia do desenvolvimento que se proponha a investigar aspectos neuropsicológicos de crianças com alterações no neurodesenvolvimento que fazem uso de instrumentos ainda não padronizados e validados para o Brasil deve ser realizada, porém, com muita cautela. Nos relatos de tais pesquisas, devem ser mencionados os pontos fortes e as fragilidades dos resultados e das interpretações da própria avaliação realizada, bem como devem ser tomados alguns cuidados éticos já discutidos no que tange à devolução de tais avaliações.

\section{Estudos de caso e as interfaces entre a Psicologia do desenvolvimento e a clínica}

O compartilhamento de experiências profissionais relatadas através de estudos de caso é uma importante estratégia de divulgação de conhecimentos (Goldim \& Fleck, 2010). O estudo de caso permite estudar o ser humano em profundidade, de maneira holística e menos fragmentada, um dos motivos pelo qual parece adequar-se a estudos clínicos (Berríos \& Lucca, 2006). Embora alguns autores questionem o uso do estudo de caso para sustentar uma conceitualização teórica, Freud (1893/1994) já se utilizou desse recurso nos primórdios da psicanálise, a fim de exemplificar a abordagem psicanalítica. Da mesma forma, ao se recorrer a Popper (Chalmers, 1993), pode-se verificar que a construção do conhecimento se dá no confronto da experiência, que pode refutar ou corroborar as hipóteses teóricas, ou seja, embora inúmeros casos não comprovem uma teoria, um único caso como contraexemplo pode levar à reavaliação de uma teoria.
A Psicologia do desenvolvimento tem como um de seus objetivos investigar a estabilidade e a mudança ao longo do ciclo vital, tanto para explorar os marcos de referência como para examinar pormenorizadamente as diferenças individuais (Belsky, 2010). Ao fazer isso, aproxima-se também dos estudos em Psicologia clínica, que podem visar tanto ao processo de mudança como aos principais resultados alcançados por indivíduos ou grupos de pacientes (Brum et al., 2012; Minuchin, 1985). Mais especificamente, pode-se considerar que ambas as abordagens tendem a tomar a família como um dos primeiros focos para compreender os indivíduos e seus comportamentos. Além disso, ambas as áreas empregam o estudo de caso como uma estratégia de produção de conhecimento.

No entanto, embora reconhecendo a importância dos estudos de caso em Psicologia, alguns dilemas éticos se impõem para a sua realização. Por exemplo, nem sempre é possível se fazer uma escolha a priori de que caso será usado para fins de publicação, pois tal escolha pode surgir a partir de alguma atividade clínica que envolve a prática profissional, não necessariamente uma pesquisa prospectiva. Isso inviabiliza a submissão do estudo a um Comitê de Ética em Pesquisa (CEP), já que não havia inicialmente nem mesmo a intenção de se fazer a pesquisa envolvendo o caso. Nessa situação, o relato de caso individual seria escolhido, por exemplo, pelo seu potencial de aprendizado (Stake, 1994) e não necessitaria da avaliação de um Comitê de Ética, já que esse tipo de relato faz parte da prática profissional (Goldim \& Fleck, 2010) e constitui ferramenta importante para o desenvolvimento da profissão (Conselho Federal de Psicologia - CFP, 2005). Assim, seria necessário apenas que o participante assinasse um Termo de Consentimento Livre e Esclarecido, nos moldes já mencionados na seção sobre o uso do TCLE. 
Entretanto, para Goldim e Fleck (2010), estudos que utilizem mais de três casos individuais devem ser encaminhados para avaliação de um comitê, pois são considerados estudos em série, o que configura atividade de pesquisa. Essa ressalva quanto ao número de casos precisa ser melhor discutida, pois é possível que, na sua prática, o profissional em Psicologia possua inúmeros casos que poderiam ser retratados em um estudo, e não há razão para que isso não possa ser feito. Afinal, como profissional, espera-se que ele contribua para o avanço da Psicologia, inclusive fazendo pesquisas (CFP, 2005). Contudo, muitas dessas investigações são realizadas inicialmente como parte de sua atuação profissional, por demandas institucionais onde atua (ex. escolas, clínicas, empresas), visando a conhecer um fenômeno para melhor intervir. Nesse contexto, não há necessariamente, de antemão, o interesse em publicar essas investigações. Mas, caso elas sejam interessantes e relevantes, não se pode impedir que sejam publicadas por não terem sido submetidas a um CEP. Nesse caso, pode-se questionar: o que vale mais o direito individual (caso) ou a demanda de uma categoria profissional visando ao avanço científico? Na tentativa de resolver a questão, uma declaração de cada participante e da instituição aceitando que os dados sejam usados em um estudo e nas publicações dele derivadas talvez atendam adequadamente aos preceitos éticos. Contudo, entende-se que ainda não há consenso para essa questão e que uma reflexão mais aprofundada precisa ser feita antes que diretrizes gerais possam ser estabelecidas. Na ausência disso, solicitar orientação a um CEP é o mais recomendável.

Outro desafio ético, após a escolha do caso, refere-se a sua descrição. Ao descrever o caso em estudo, é importante que, além da omissão ou da substituição do nome ou das iniciais do nome do participante, se omitam datas importantes, como, por exemplo, a data de nascimento, excetuando-se o ano, quando pertinente, a origem geográfica e alguns dados sociodemográficos, como profissão, para a proteção da privacidade e do anonimato do participante (Goldim \& Fleck, 2010). Aqui surge uma questão nos estudos de casos realizados com famílias: o que fazer quando se entrevistam separadamente membros da mesma família, por exemplo, quando cada membro de um casal é entrevistado individualmente em uma pesquisa sobre conjugalidade? Embora ambos tenham consentido em participar da pesquisa, ainda assim é importante primar pela privacidade de cada membro. Podemos omitir todas as informações pessoais destacadas acima, mas é provável que o próprio participante se reconheça e, a partir disso, identifique dados dos outros membros de sua família, que, em princípio, não deveriam ser revelados. Esse conflito ainda não tem uma solução clara. Francisconi e Goldim (1998) também problematizam essa questão, mas os autores se referem especialmente ao acesso aos prontuários em terapia de família pelos próprios clientes, e não à situação de pesquisa, e sugerem que haja uma combinação prévia com a família quanto a esse aspecto. Uma possível solução seria omitir, além dos dados sociodemográficos, as vinhetas de estudos qualitativos, ou então mostrar previamente o manuscrito aos participantes, para que eles autorizassem sua publicação.

Além da identificação de dados e vinhetas que podem ser atribuídas a um ou outro membro da família, também devem ser consideradas as relações, conexões e interpretações que o pesquisador faz com os dados coletados, que podem levar a um mal-estar entre os participantes. Afinal, embora a sua vida seja conhecida por eles mesmos, o mais chocante podem ser as interpretações acerca do que relataram. Nesse sentido, o pesquisador em Psicologia precisa ser bastante cauteloso, buscando sempre relativizar suas afirmações, tanto por respeito aos familiares que poderão 
vir a conhecer o estudo como pelo fato de que na ciência não existem verdades absolutas, e nós, pesquisadores, somos seus eternos buscadores, como bem nos lembra Popper (Chalmers, 1993).

Outra questão referente ao estudo de caso diz respeito à possibilidade de o pesquisador ser também o psicoterapeuta, $\mathrm{o}$ que pode gerar outros dilemas. Ao discorrer sobre a ética na Medicina em países em desenvolvimento, Cabral, Schindler e Abath (2006) relembraram o tema da inversão da relação entre o paciente e o pesquisadorclínico em contexto de pesquisa. Embora os autores tenham se referido à Medicina, pode-se verificar que a presença desse dilema ético está presente em várias áreas da saúde humana, incluindo a Psicologia, pois, em contexto de pesquisa clínica, não apenas o participante tem suas necessidades satisfeitas ao buscar uma psicoterapia, por exemplo, como o pesquisador-clínico pode considerar que esse participante também satisfaz as necessidades e os interesses da ciência. Por isso, tal inversão deve ser explicitada, a fim de que o participante tenha seu atendimento assegurado acima dos interesses do pesquisador.

Nesse sentido, em alguns projetos de pesquisa (referência omitida para preservar autoria), as pesquisadoras-psicoterapeutas apenas realizaram a análise dos casos para a elaboração de suas teses após a finalização da psicoterapia com cada caso. Esse procedimento seguiu a sugestão de Giami (2004), que indica que não se pode conduzir concomitantemente pesquisa e psicoterapia com a mesma pessoa. Segundo esse autor, na pesquisa clínica, o a posteriori da coleta do material permite suspender a relação com o sujeito quando sua problemática é discutida à luz das hipóteses da pesquisa. Embora se entenda que, em outras situações, pode ser necessário contemplar pesquisa e psicoterapia no mesmo momento, deve-se considerar que tal fato pode tornar complexo o processo em termos de seus objetivos terapêuticos, que podem não ser os mesmos do pesquisador. Nesse sentido, endossa-se a sugestão de Giami, pois favorece maior possibilidade de descontaminar o objetivo terapêutico dos objetivos da pesquisa. Enfim, estar atento e priorizar o bem-estar do participante é algo que o pesquisador deve considerar independentemente do desenho metolodológico e da estratégia de pesquisa utilizada, a fim de que possa efetivamente examinar as mudanças ao longo do ciclo vital, ou aquelas resultantes de um contexto clínico.

\section{Considerações finais}

O objetivo do presente estudo foi discutir alguns dilemas éticos da pesquisa em Psicologia do desenvolvimento. As reflexões apresentadas revelam que, apesar dos avanços na regulamentação, a prática da pesquisa, e especialmente sua dimensão ética, impõe constantemente novos desafios. A análise dos dilemas apresentados revela que, em muitos casos, ainda não há soluções ou respostas satisfatórias, e que, portanto, o bom senso pautado no conhecimento da legislação vigente e na prática em pesquisa muitas vezes precisa ser priorizado. Como orientação geral, é sempre importante evitarse a tendência a racionalizar e a subestimar os riscos de nossas pesquisas e de superestimar seus benefícios, assim como a ideia de que "os meios justificam os fins" (Barker et al., 1995). Também é importante lembrar que, de modo geral, existe um desnível de poder entre o pesquisador e o eventual participante, especialmente em instituições, sejam elas públicas, sejam privadas, o que pode provocar no participante a impressão de que a rejeição de um convite para participar de uma pesquisa pode prejudicar seu tratamento ou reduzir a atenção dos profissionais para o seu problema.

Desse modo, percebe-se que o mais importante é que essa atitude de reflexão e de questionamento ético sempre esteja presente na concepção das nossas pesquisas, mesmo 
quando é necessário usar o bom senso frente à ausência de uma legislação específica, como apontado em algumas das situações tratadas no presente estudo. A partir de uma postura crítica, reflexiva e comprometida que cada pesquisador deve assumir diante dos aspectos éticos da sua pesquisa, muitas ideias novas poderão surgir, e assim contribuir para o avanço nos debates sobre questões polêmicas e de difícil manejo.

É importante destacar que as questões apontadas no presente estudo refletem apenas algumas das inquietações que afligem e instigam os pesquisadores, não contemplando a diversidade de desafios e dilemas a serem analisados. Outros questionamentos, como, por exemplo, a possibilidade de recompensar financeiramente os participantes (Cabral et al., 2006), assim como as dificuldades geradas por projetos que necessitam de aprovação de mais de um Comitê de Ética, precisam ser ainda discutidas.

Para tanto, é fundamental que se promova esse tipo de discussão e que os dilemas emergentes e suas possíveis alternativas de solução sejam compartilhadas e disseminadas tanto entre os pesquisadores como na comunidade. A história das últimas décadas nos mostra que considerar a discussão dessas questões éticas como tarefa apenas dos pesquisadores nem sempre permitiu que a prática da pesquisa avançasse rumo a padrões éticos mais elevados. Por isso, é muito importante que a comunidade esteja presente nesses debates, como já está, até certo ponto, nos Comitês de Ética existentes em nosso país. 


\section{Patrícia Alvarenga}

Doutora em Psicologia do Desenvolvimento pela Universidade Federal do Rio Grande do Sul e professora do Programa de Pós-Graduação em Psicologia da Universidade Federal da Bahia, Salvador - BA - Brasil.

E-mail: palvarenga66@gmail.com

\section{Cesar Augusto Piccinini}

Doutor em Psicologia pela University College London (Inglaterra), professor do Programa de Pós-Graduação em Psicologia do Desenvolvimento da Universidade Federal do Rio Grande do Sul. Porto Alegre - RS - Brasil. E-mail: piccinini@portoweb.com.br

\section{Daniela Centenaro Levandowski}

Doutora em Psicologia do Desenvolvimento (UFRGS), com Pós-doutorado em Psicologia (PUCRS).

Docente do Departamento de Psicologia e do Programa de Pós-Graduação em Ciências da Saúde da Universidade Federal de Ciências da

Saúde de Porto Alegre (UFCSPA), Porto Alegre - RS - Brasil.

E-mail: d.cl@terra.com.br'

\section{Giana Bitencourt Frizzo}

Doutora em Psicologia pela Universidade Federal do Rio Grande do Sul, professora do Departamento de Psicologia do Desenvolvimento e da Personalidade da Universidade Federal do Rio Grande do Sul, Porto Alegre - RS - Brasil.

E-mail: gifrizzo@hotmail.com

\section{Angela Helena Marin}

Doutora em Psicologia pela Universidade Federal do Rio Grande do Sul, professora do Curso de Psicologia da Universidade Luterana do Brasil, Canoas - RS - Brasil.

E-mail: ahmarin@hotmail.com

\section{Pompéia Villachan-Lyra}

Doutora em Psicologia Cognitiva pela Universidade Federal de Pernambuco (UFPE), professora do Departamento de Educação e coordenadora do Núcleo de Investigação em Neuropsicologia, Aprendizagem e Primeira Infância (NINAPI/UFRPE) da Universidade Federal Rural de Pernambuco, UFPE, Recife - PE - Brasil. E-mail: lyrapomp@gmail.com

\section{Endereço para envio de correspondência:}

Universidade Federal da Bahia - Instituto de Psicologia. Rua Aristides Novis, 197. CEP: 40210-730. Bairro: Federação. Salvador/BA.

Recebido 07/11/2011, 1a Reformulação 04/06/2012, Aprovado 15/06/2012.

Referências
Alvarenga, P., Dazzani, M. V. M., Lordelo, E. R., Alfaya, C. A. S, \& Piccinini, C. A. (2007). Determinantes sociodemográficos, familiares e individuais do comportamento anti-social na infância. Projeto de pesquisa não publicado. Instituto de Psicologia, Universidade Federal da Bahia, Salvador, BA.

Barker, C., Pistrang, N., \& Elliot, R. (1995). Research methods in clinical and counselling psychology. New York: Wiley.

Belsky, J. (2010). Desenvolvimento humano: experienciando o ciclo da vida (D. Bueno, trad.). Porto Alegre: Artmed (Trabalho original publicado em 2010).

Berríos, R., \& Lucca, N. (2006). Qualitative methodology in counseling research: Recent contributions and challenges for a new century. Journal of Counseling and Development, 84, 174-186.

Binder, L., \& Thompson, L. (1995). The ethics code and neuropsychological assessment practices. Archives of Clinical Neuropsychology, 10(1), 27-46.

Brasil. (1990). Presidência da República. Casa Civil. Subchefia para Assuntos Jurídicos. Estatuto da Criança e do Adolescente. Lei 8069 de 13 de julho de 1990. Recuperado em 19 de outubro, 2011, de http://www.planalto.gov.br/ccivil_03/leis/ L8069.htm

Brasil. (1996). Ministério da Saúde. Conselho Nacional de Saúde.
Resolução no 196. Recuperado em 19 julho, 2011, de http:// conselho.saude.gov.br/resolucoes/reso_96.htm.

Brasil. (1999). Ministério da Saúde. Secretaria de Políticas de Saúde. Coordenação Nacional de DST e AIDS. Diretrizes dos Centros de Testagem e Aconselhamento (CTA) - Manual. Brasília. Recuperado em 19 julho, 2011, de http://bvsms. saude.gov.br/bvs/publicacoes/diretrizes_cta.pdf.

Brasil (2002). Código Civil Brasileiro. Recuperado em 19 julho, 2011, de http://www.planalto.gov.br/ccivil_03/leis/2002/ L10406.htm

Brum, E. H. M., Frizzo, G. B., Gomes, A. G., Silva, M. R., Souza, D. D., \& Piccinini, C. A. (2012). Evolução dos modelos de pesquisa em psicoterapia. Estudos de Psicologia, 29, 259-269.

Cabral, M. M. L., Schindler, H. C., \& Abath, F.G.C. (2006). Regulamentações, conflitos e ética da pesquisa médica em países em desenvolvimento. Revista de Saúde Pública, 40(3), 521-527

Chalmers, A. F. (1993). O que é ciência afinal? São Paulo: Brasiliense.

Conselho Federal de Psicologia - CFP. (2000). Resolução $n^{\circ}$ 016/2000. Recuperado em 19 julho, 2011, de http://site.cfp. org.br/wp-content/uploads/2000/12/resolucao2000_16.pdf

Conselho Federal de Psicologia - CFP. (2005). Código de ética Profissional. Recuperado em 19 julho, 2011, dehttp://www. 
pol.org.br/pol/cms/pol/legislacao/codigo_etica/

Dessen, M. A., Avelar, L. P., \& Dias, R. L. S. (1998). Questões éticas na pesquisa com famílias. Paidéia, 8, 169-180.

Figueiredo, B. (2001). Maternidade na adolescência: do risco à prevenção. Revista Portuguesa de Psicossomática, 3(2), 221-237.

Flicker, S., \& Guta, A. (2008). Ethical approaches to adolescent participation in sexual health research. Journal of Adolescent Health, 42, 3-10.

Francisconi, C. F., \& Goldim, J. R (1998). Aspectos bioéticos da confidencialidade e privacidade. In S. I. F. Costa, V. Garrafa, \& G. Oselka (Orgs.). Iniciação à bioética. Brasília, DF: Conselho Federal de Medicina.

Freud, S., \& Breuer, J. (1994). Sobre el mecanismo psíquico de fenómenos histéricos: comunicación preliminar. Estudios sobre la histeria. In S. Freud, Obras completas (Vol. 2). Buenos Aires: Amorrortu. (Trabalho original publicado em 1893).

Frizzo, G. B., \& Piccinini, C. A. (2005). Interação mãe-bebê em contexto de depressão materna: Aspectos teóricos e empíricos. Psicologia em Estudo, 10(1), 47-55.

Giami, A. (2004). Pesquisa em psicologia clínica ou pesquisa clínica. In C. R. D'Allones, C. Assouly-Piquet, F. B., Slama, A., Blanchet, O., Douville, A., Giami, K. Nguyen, M., Plaza, \& C. Samalin-Amboise. Os procedimentos clínicos nas ciências humanas - documentos, métodos, problemas (pp.35-52). São Paulo: Casa do Psicólogo.

Goldim, J. R. (1997). Pesquisa em saúde: leis, normas e diretrizes. Porto Alegre: HCPA.

Goldim, J. R. (2006). O consentimento informado numa perspectiva além da autonomia. In R. T. Souza (Org.). Ciência e ética: os grandes desafios (pp.45-64). Porto Alegre: EDIPUCRS.

Goldim, J. R., \& Fleck, M. (2010). Ética e publicação de relatos de caso individuais. Revista Brasileira de Psiquiatria, 32(1), 1-2.

Goldim, J. R., Pithan, C. F., Oliveira, J. G., \& Raymundo, M. M. (2003). O processo de consentimento informado livre e esclarecido em pesquisa: uma nova abordagem. Revista da Associação Médica Brasileira, 49(4), 372-374.

Guariglia, F., Bento, S. F., \& Hardy, E. (2006). Adolescentes como voluntários de pesquisa e consentimento livre e esclarecido: Conhecimento e opinião de pesquisadores e jovens. Cadernos de Saúde Pública, 22(1), 53-62.

Haase, V. G., \& Rothe-Neves, R. (2007). Ética e pesquisa comportamental. In D. Guilhem, Diniz, D., \& F. Zicker (Orgs.). Pelas lentes do cinema: bioética e ética em pesquisa (pp.181-208). Brasilia, DF: LetrasLivres / Editora da UnB.

Hazin, I., Garcia, D., Gomes, E., \& Lemos, C. (no prelo). Aspectos neuropsicológicos do desenvolvimento infantil. In K. Moutinho, P. Villachan-Lyra \& A. Santa-Clara. Novas tendências_em Psicologia do Desenvolvimento (pp. 157-175).

Holder, A. R. (2008). Research with adolescents: Parental involvement required? Journal of_Adolescent Health, 42, 1-2.

Koller, S. H. (2008). Ethics in research with human beings: Some issues about Psychology. Ciência \& Saúde Coletiva, 13(2), 399-406
Levandowski, D. C.; Castoldi, L.; Canavarro, M. C., \& Pereira, M. D. (2010). Avaliação e Intervenção com Mães Adolescentes Soropositivas: Focalizando a Saúde Mental, a Adesão ao Tratamento e a Relação com o Bebê. Projeto de Pesquisa não publicado. UFCSPA, Porto Alegre, RS.

Levandowski, D. C. (2008). Avaliação de Aspectos Emocionais de Adolescentes do Vale do Rio dos Sinos na Transição para a Parentalidade: Um Estudo Longitudinal. Projeto de Pesquisa não publicado. UNISINOS/UFCSPA, Porto Alegre, RS

Libório, R. M. C., \& Castro, B. M. de. (2010). Abuso, exploração sexual e pedofilia: as intrincadas relações entre os conceitos e o enfrentamento da violência sexual contra crianças e adolescentes. In M. A. Ungaretti (Org.). Crianças e adolescentes: direitos, sexualidades e reprodução (pp. 19-42). São Paulo: ABMP.

Miller, S. A. (2007). Developmental research methods. Thousand Oaks: Sage Publication Inc.

Minuchin, P. (1985). Families and individual development: Provocations from the field of family therapy. Child Development, 56, 289-302.

Muszkat, M. (2006). Desenvolvimento e neuroplasticidade. In C. B. Mello, M. C., Miranda, \& M. Muszkat (Orgs.). Neuropsicologia do desenvolvimento: conceitos e abordagens (pp. 26-45). São Paulo: Memnon.

Neiva-Silva, L., Lisboa, C. S. de M., \& Koller, S. H. (2005). Bioética na pesquisa com crianças e adolescentes em situação de risco: dilemas sobre o consentimento e a confidencialidade. Jornal Brasileiro de Doenças Sexualmente Transmissíveis, 17(3), 201-206.

Organização das Nações Unidas. (2011). Sexual and reproductive health - ethical issues: Reproductive health involving adolescents. Recuperado em 19 julho, 2011, de http://www.who.int/ reproductivehealth/topics/ethics/adolescents_guide_serg/en/.

Piccinini, C. A, Lopes, R.C.S., Marin, A.H., Carvalho, F.T., Henn, C.G., Diehl, A.P., Dias, A.C.G. \& Souza, D.S.(2008). Aspectos Biopsicossociais da Gestação na Adolescência. Projeto de pesquisa não publicado. Instituto de Psicologia, Universidade Federal do Rio Grande do Sul, Porto Alegre, RS.

Piccinini, C., Prado, L., Lopes, R., Schwengber, D., Alfaya, C., Frizzo, G., Mayor, I., \& Silva, M. (2003). O impacto da psicoterapia para a depressão materna e para a interação pais-bebê: Estudo longitudinal do nascimento ao segundo ano de vida do bebê. Projeto de pesquisa não publicado. Instituto de Psicologia, Universidade Federal do Rio Grande do Sul, Porto Alegre, RS.

Santelli, J. S., Rogers, A. S., Rosenfeld, W. D., DuRant, R. H., Dubler, N., Morreale, M., English, A., Lyss, S., Winberly, Y., \& Schissel, A. (2003). Guidelines for adolescent health research. A position paper of the Society for Adolescent Medicine. Journal of Adolescent Health, 33, 396-409.

Stake, R. E. (1994). Case studies. In N. Denzin \& Y. Lincoln (Eds.). Handbook of qualitative research (pp. 236-247). London: Sage.

Schwengber, D. D. S., \& Piccinini, C. A. (2003). O impacto da depressão pós-parto para a interação mãe-bebê. Estudos de Psicologia, 8(3), 403-411.

Taquette, S. R., Vilhena, M. M. de, Silva, M. M., \& Vale, M. P. (2005). Conflitos éticos no atendimento à saúde de adolescentes. Cadernos de Saúde Pública, 21(6), 1717-1725. 